Mechanism of giant enhancement of light emission from $\mathrm{Au} / \mathrm{CdSe}$ nanocomposites

This article has been downloaded from IOPscience. Please scroll down to see the full text article.

2007 Nanotechnology 18415707

(http://iopscience.iop.org/0957-4484/18/41/415707)

The Table of Contents and more related content is available

Download details:

IP Address: 140.112.113.225

The article was downloaded on 26/06/2009 at 10:58

Please note that terms and conditions apply. 


\title{
Mechanism of giant enhancement of light emission from Au/CdSe nanocomposites
}

\author{
Ya-Ping Hsieh ${ }^{1}$, Chi-Te Liang ${ }^{1}$, Yang-Fang Chen ${ }^{1}$, Chih-Wei Lai ${ }^{2}$ \\ and Pi-Tai Chou ${ }^{2}$ \\ ${ }^{1}$ Department of Physics, National Taiwan University, Taipei 106, Taiwan \\ ${ }^{2}$ Department of Chemistry, National Taiwan University, Taipei 106, Taiwan \\ E-mail: ctliang@phys.ntu.edu.tw and yfchen@phys.ntu.edu.tw
}

Received 22 June 2007, in final form 1 August 2007

Published 17 September 2007

Online at stacks.iop.org/Nano/18/415707

\begin{abstract}
Based on the enhanced electron-hole recombination rate generated by surface plasmon (SP) waves of Au nanoparticles (NPs) and electrons transferred from CdSe quantum dots (QDs) to Au NPs, we propose a mechanism to elucidate the luminescent behavior in $\mathrm{Au}$ and $\mathrm{CdSe}$ nanocomposites. With our proposed model, the enhancement of the spectrally integrated PL intensity can be manipulated by up to a factor of $\sim 33$, the largest value ever reported. Our study can be used to clarify the ambiguity in controlling the light emission enhancement and quenching of semiconductor nanocrystals coupled with the SP waves of metal NPs. It should be very useful for the creation of highly efficient solid-state emitters.
\end{abstract}

(Some figures in this article are in colour only in the electronic version)

\section{Introduction}

As a result of the quantum-confinement effect, semiconductor quantum dots (QDs) can emit light fine-tuned in the visible spectrum and beyond [1]. Because of their spectral tunability, large quantum efficiency, and photo-stability, semiconductor QDs have been extensively studied for a wide variety of potential applications such as light emitters [2, 3], fluorescent labels $[4,5]$ and photocatalysis [6].

Surface enhanced Raman scattering has been exploited as a basis for ultra-sensitive analyses in molecular science, biology and medicine [6-9]. In particular, recent advances in metal nanostructures have initiated an entirely new branch of photonics based on surface plasmons [8]. Intuitively, one expects that many interesting physical phenomena could be observed if the characteristic behavior of metal nanoparticles (surface plasmon) and semiconductor QDs (strong luminescence) can be integrated [10]. Consequently there have been numerous studies on the emission enhancement in nanocrystal chromophores under different environments [6, 10-12]. However, quenching rather than enhancement of the QD emission by metal nanoparticles (NPs) is often observed [6]. Even though there has been some success, the optical emission enhancement via surface plasma-quantum dots (SP-QDs) coupling is rather limited ( $<5$ fold) [10-12]. It is believed that the unambiguous determination of the mechanism for the observed emission enhancement and quenching will yield new insights into the basic aspects of the interaction between the electromagnetic waves and the charged particles in metal-semiconductor nanocomposites. In addition, it will allow us to optimize the optical emission enhancement and to better implement nanocomposites in biological and solid-state device applications. In this paper, a unifying model is proposed to resolve the physical origin of the optical enhancement and quenching observed in $\mathrm{Au} / \mathrm{CdSe}$ nanocomposites, which consequently offers excellent prospects for controlling the emission behavior in semiconductor QDs. For example, by varying the size of the CdSe QDs, and the concentration of the added Au NPs, we are able to achieve an enhancement ratio of spectrally integrated photoluminescence (PL) intensity of up to $\sim 33$. Our results therefore provide a great opportunity for creating highly efficient optoelectronic devices.

\section{Experiments}

Au colloids were synthesized based on the method described in [13]. CdSe QDs with different radii were synthesized and purified according to the procedure reported in [14]. Equal amounts of CdSe powder were stuffed into cylindrical cavities of $0.3 \mathrm{~cm}$ in diameter and $0.15 \mathrm{~cm}$ in depth, and equal numbers 

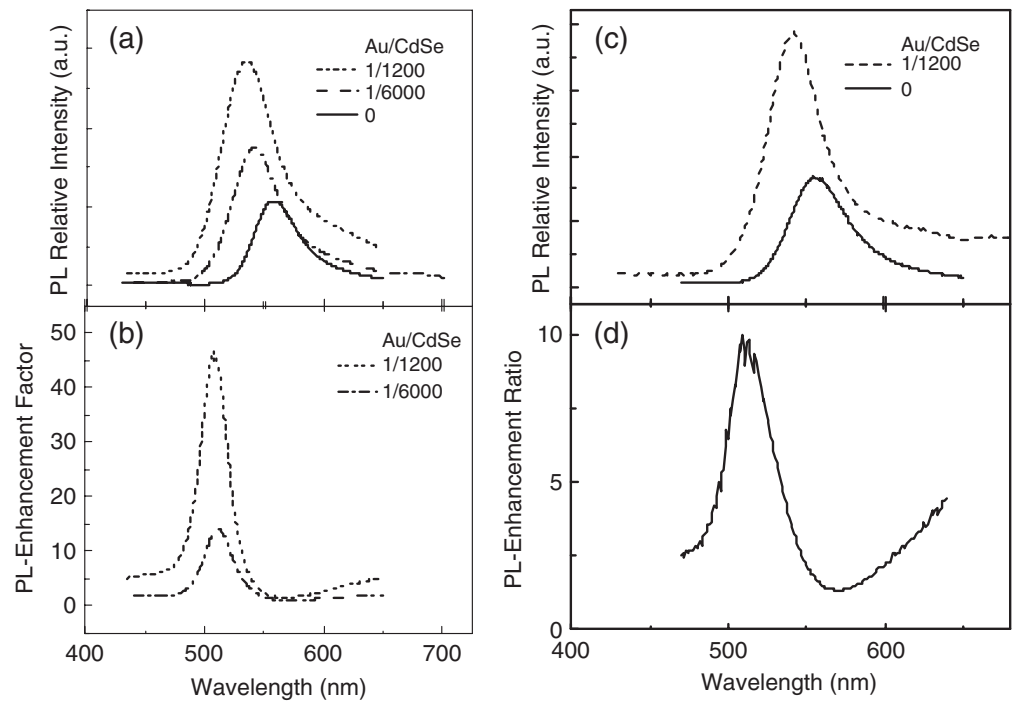

Figure 1. (a) PL spectra of CdSe quantum dots upon adding 18-nm-diameter Au NPs, with the Au/CdSe NP ratio ranging from 0 to $1 / 1200$. (b) PL enhancement spectrum $R(\lambda)$ for the same samples as in (a). (c) PL spectra of CdSe quantum dots upon adding different concentrations of 3-nm-diameter Au NPs. (d) PL enhancement spectrum $R(\lambda)$ for the same sample as in (c).

of drops of Au colloids with different molar concentrations were added. The samples were then allowed to dry completely, before room temperature PL data were recorded. The PL spectra were measured using a SPEX Flurolog-2 instrument equipped with a double emission monochromator and an R928 photomultiplier tube. Excitation was provided by an $\sim 450 \mathrm{~W}$ Xe lamp whose output was focused into a $0.22 \mathrm{~m}$ monochromator to provide appropriate wavelength selection. Unless otherwise stated, the photoexcitation wavelength was fixed at $400 \mathrm{~nm}$.

\section{Results and discussion}

Figure 1(a) shows the PL spectra of CdSe QDs before and after adding 18-nm-diameter Au NPs with two different $\mathrm{Au} / \mathrm{CdSe}$ nanoparticle ratios of 1/1200 and 1/6000. The PL spectrum for CdSe QDs peaks at $555 \mathrm{~nm}$. As shown in figure 1(a), the peaks of the PL spectra for CdSe QDs are blue-shifted (66 and $98 \mathrm{meV}$ ) after the addition of Au NPs. In order to obtain the PL enhancement spectra $R(\lambda)$, the PL spectra of CdSe QDs and Au NPs are divided by that for CdSe QDs as shown in figure 1(b). Surprisingly, we found that the maxima of the PL-enhancement ratios are always at $\sim 510 \mathrm{~nm}$, independent of the amount of the blue-shift in the PL spectra. The PL enhancement spectra have very narrow line widths, and are confined mostly in the region between 470 and $530 \mathrm{~nm}$, which corresponds to the transition energy of excited states in CdSe QDs [15]. Figure 1(c) shows the PL spectra for CdSe QDs upon adding 3-nm-diameter Au NPs. As shown in figure 1(d), the PL-enhancement ratio also exhibits a similar distribution between 470 to $530 \mathrm{~nm}$, and peaks at $510 \mathrm{~nm}$. All of the data shown in figure 1 suggest that the $R(\lambda)$ spectrum is an intrinsic property of CdSe QDs, because they show very little dependence on the size of the added Au NPs.

To further study the physical origin of the observed PL enhancement, we have measured the PL spectra for CdSe QDs with different sizes, before and after the addition of 3-nmdiameter $\mathrm{Au}$ NPs with a $\mathrm{Au} / \mathrm{CdSe}$ particle ratio of $1 / 240$, as shown in figure 2(a). Before the addition of Au NPs, for simplicity, the CdSe QDs with a PL peak around $555 \mathrm{~nm}$ are denoted as CdSe 555, and so on. We note that the PL intensity can be either enhanced or reduced, depending on the size of the CdSe QDs as shown in figure 2(a). The enhancement factor distribution $R(\lambda)$ shown in figure 2(b) illustrates the dependence on the size of the CdSe QDs. We can see that at $\lambda \sim 529 \mathrm{~nm}$ the $R(\lambda)$ for sample CdSe 572 is $\sim 130$, which is the largest value ever reported. In order to compare this with the results published previously, we have calculated the enhancement ratio of the spectrally integrated PL intensity, which is as large as 33 times. This value is also much larger than previous measurements [10-12].

Before further discussion, we first examine some important properties of the added Au NPs since they drastically affect the light emission of CdSe QDs. The absorption spectrum of 3-nm-diameter Au NPs is shown in the inset of figure 2(b). We can see that the peak energy of the surface plasmon resonance of Au NPs is in excellent agreement with that of the maximum of the PL-enhancement ratio of sample CdSe 572. We also note that when the peak energy of $R(\lambda)$ for CdSe QDs gets closer to the Au SP energy, the maximum of the PL-enhancement ratio is larger. Our results thus strongly suggest that in order to have an optimized PL-enhancement ratio from $\mathrm{CdSe} / \mathrm{Au}$ nanocomposites, the energy difference between the electron and hole states in CdSe QDs has to be close to the SP energy of Au NPs.

In order to provide a thorough understanding of the mechanisms for the observed PL enhancement and quenching effects, we study the PL enhancement spectrum $R(\lambda)$ for sample CdSe 572 upon adding various concentrations of $\mathrm{Au}$ as shown in figure 3. As one can see, the PL-enhancement ratio reaches a maximum value when the $\mathrm{Au} / \mathrm{CdSe}$ nanoparticle ratio has a value of $1 / 240$. Note that the signal of the $700 \mathrm{~nm}$ band shown in figure 3 arises from defect emission [16]. 

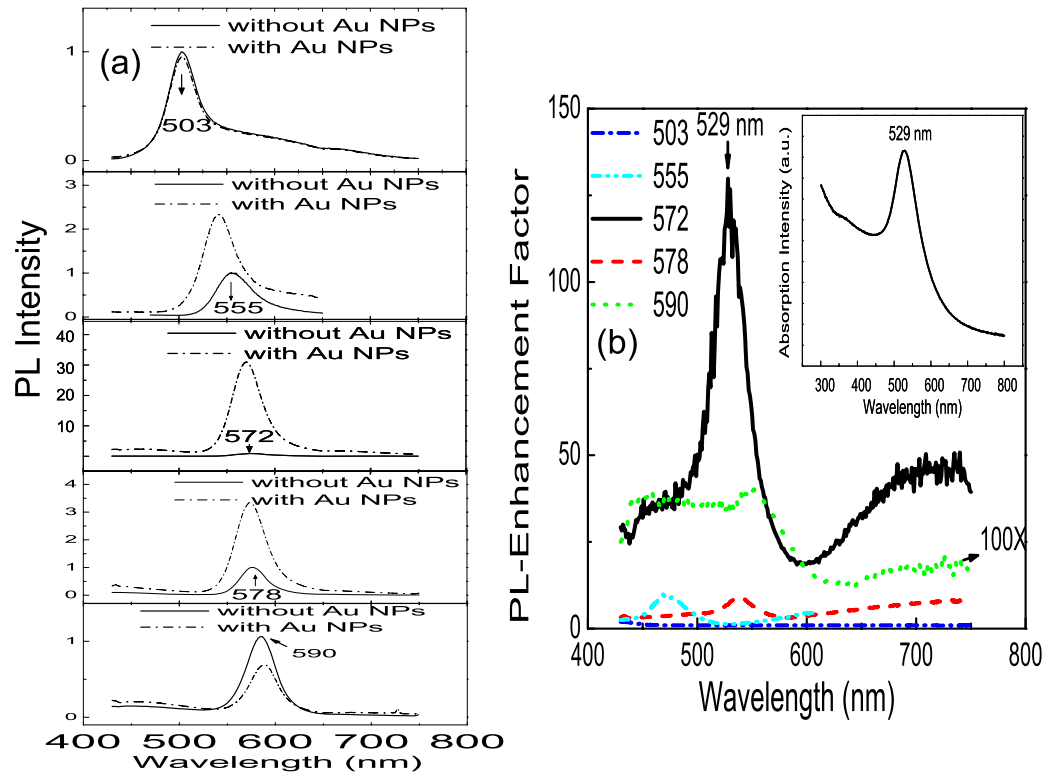

Figure 2. (a) PL spectra for CdSe quantum dots of different sizes without Au NPs (solid lines) and with 3-nm-diameter Au NPs having a $\mathrm{Au} / \mathrm{CdSe}$ NP ratio of 1/240 (dash-dot lines). The spectra are normalized to the peak value of the spectrum in the sample without Au NPs. (b) PL enhancement spectrum $R(\lambda)$ for the same samples as in (a). The inset to (b) shows the absorption spectrum for 3-nm-diameter Au NPs.

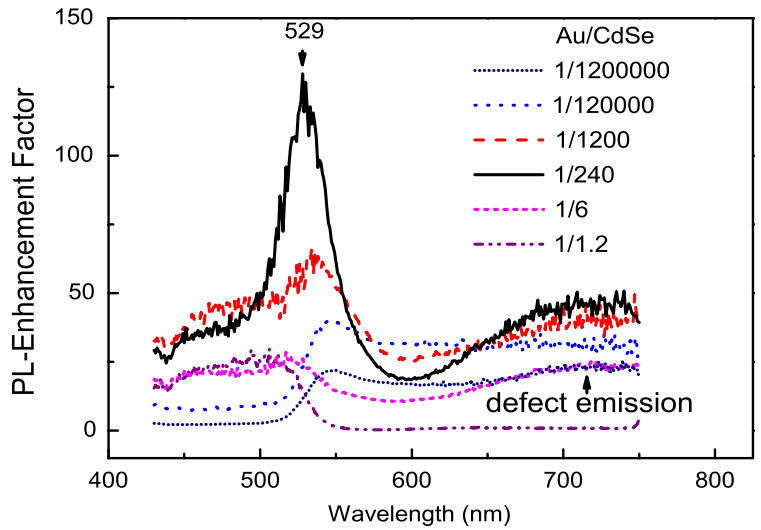

Figure 3. (a) PL-enhancement spectrum $R(\lambda)$ for sample CdSe 572 upon adding $\mathrm{Au}$ NPs with a $\mathrm{Au} / \mathrm{CdSe} \mathrm{NP}$ ratio ranging from $1 / 1200000$ to $1 / 1.2$.

We now proceed to pinpoint the key factors responsible for the enhancement and quenching of the light emission from CdSe QDs. As shown in figure 2, the size of the CdSe QDs is one of the dominant factors. According to the data shown in the inset of figure 2(b), we conclude that the size of the CdSe QDs can be used to fine-tune the effective transition energy of the CdSe QDs close to the SP energy of Au NPs. It has been proposed that the locally enhanced electromagnetic field due to the SP waves of Au NPs can result in a more effective QD excitation [11]. Therefore, through the resonant excitation by the SP waves of Au NPs, the PL emission intensity of the $\mathrm{CdSe} / \mathrm{Au}$ nanocomposites is enhanced. We then consider the influence of Au NP concentration. It is well known that the $\mathrm{SP}$-quantum well (QW) coupling increases with decreasing metal-QW distance. Here we point out that the concentration of added Au NPs can be used to determine the average separation between CdSe QDs and Au NPs. Initially, both the PL intensity and $R(\lambda)$ are expected to increase as the concentration of added Au NPs increases due to the increased local field by $\mathrm{Au}$ surface plasmons. However, the distance between CdSe QDs and Au NPs also dictates the ability to transfer electrons from the conduction band edge of CdSe QDs to the Fermi level of the NPs. It is well established that the emission quenching originates mainly from the electron transfer from semiconductor QDs to metal NPs [6]. Thus, as the concentration of Au NPs exceeds a certain threshold, CdSe QDs and Au NPs would be so close to one another that electrons can now easily transfer from QDs to Au particles, resulting in a quenching of the emission [6]. Therefore, upon reaching the maximum value, a further increase in the $\mathrm{Au}$ concentration will result in a decrease of the PL-enhancement ratio, due to the enhanced electron transfer rate from the conduction band of the CdSe QDs to the Au NPs. This behavior is clearly revealed by our experimental result as shown in figure 3 . According to our study, when we take the threshold ratio $\mathrm{Au} / \mathrm{CdSe}=1 / 240$ into account and assuming that the occupation of gold nanoparticles uniformly spreads over all of the cavity, the threshold distance between CdSe and $\mathrm{Au}$ in our experiments is estimated to be around $10 \mathrm{~nm}$, which is consistent with the results of Gueroui et al [17]. Finally, in order to clearly illustrate our proposed mechanism, a brief diagram for the enhancement and quenching of the emission is shown in figure 4. It shows that the optical enhancement and quenching are a consequence of the interplay between electron-hole pairs generated by the SP waves of Au NPs and electron transfer from CdSe QDs to Au NPs.

\section{Conclusion}

In summary, it was found that the enhancement and quenching of the emission from composites of CdSe QDs and Au NPs are a result of the interplay between the increased QD excitation and electron transfer from CdSe QDs to the Fermi level of $\mathrm{Au}$ NPs. Based on our proposed mechanism, through 
(a) enhancement process

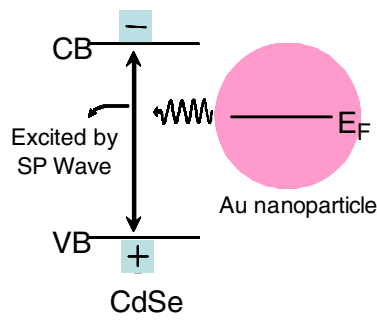

(b) quenching process

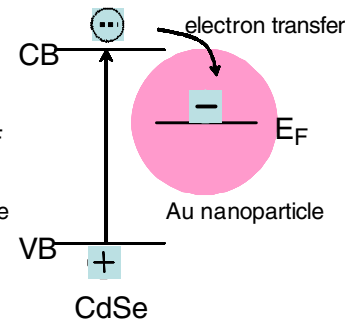

Figure 4. A schematic diagram showing the PL (a) enhancement and (b) quenching processes from $\mathrm{CdSe} / \mathrm{Au}$ nanocomposites with the variation of $\mathrm{CdSe}-\mathrm{Au}$ distance.

careful manipulation, the PL-enhancement ratio of spectrally integrated intensity can be optimized up to $\sim 33$, which is the largest value ever reported to date. Our experimental result therefore clarifies the ambiguity in controlling the emission enhancement of semiconductor nanocrystals coupled with surface plasmon waves, and it should be very important for future application in optoelectronic devices.

\section{Acknowledgments}

This work was funded by the NSC and MOE of the Republic of China.

\section{References}

[1] Marray C B, Kagan C R and Bawendi M G 2000 Annu. Rev. Mater. Sci. 30545

[2] Tsutsui T 2002 Nature 420752

[3] Colvin V L, Schlamp M C and Alivisatos A P 1994 Nature 370354

[4] Chan W C W and Nie S 1998 Science 2812016

[5] Bruchez M, Moronne M, Gin P, Weiss S and Alivisatos A P 1998 Science 2812013

[6] Nikoobakht B, Burda C, Braun M, Hun M and El-Sayed M A 2002 Photochem. Photobiol. 75591

[7] Cao Y C, Jin R and Mirkin C A 2002 Science 2971536

[8] Barnes W L, Dereux A and Ebbeson T W 2003 Nature 424824

[9] Werner J H, Kolodinski S and Queisser H J 1994 Phys. Rev. Lett. 723851

[10] Shimizu K T, Woo W K, Fisher B R, Eisler H J and Bawendi M G 2002 Phys. Rev. Lett. 89117401

[11] Kulakovich O, Strekal N, Yaroshevich A, Maskevich S, Gaponenko S, Nabiev I, Woggon U and Artemyev M 2002 Nano Lett. 21449

[12] Lee J, Govorov A O, Dulka J and Kotov N A 2004 Nano Lett. 42323

[13] Jana N R, Gearheart L and Murphy C J 2001 Langmuir 176782

[14] Qu L H and Peng X G 2002 J. Am. Chem. Soc. 1242049

[15] Klimov V I, McBranch D W, Leatherdale C A and Bawendi M G 1999 Phys. Rev. B 6013740

[16] Poles E, Selmarten D C, Mićić O I and Nozik A J 1999 Appl. Phys. Lett. 75971

[17] Gueroui Z and Libchaber A 2004 Phys. Rev. Lett. 1.93166108 